\title{
Magnetic Activity of Pre-Main Sequence Stars
}

\author{
Eric D. Feigelson \\ Department of Astronomy $\&$ Astrophysics, \\ Pennsylvania State University, 525 Davey Laboratory, \\ University Park PA 16802 USA
}

\begin{abstract}
.
I review here recent advances in our understanding of magnetic activity in pre-main sequence (PMS) protostars and T Tauri stars. Results are based on recent imaging, spectroscopic and temporal studies of nearby star forming regions from the Chandra $X$-ray Observatory and $X M M-N e w t o n$, including a first look at an ultradeep Chandra exposure of the Orion Nebula Cluster.

Pre-main sequence stars exhibit a high level of X-ray emission dominated by a bewildering variety of magnetic reconnection flares. Activity is linked to bulk stellar properties $-L_{b o l}$, mass, surface area or volume rather than rotation. This suggests that dynamo processes in deeply convective PMS stars may fundamentally differ from the tachocline dynamo operating in main sequence stars.

$\mathrm{X}$-rays and $\mathrm{MeV}$ particles from magnetic flares will affect the circumstellar environment in PMS systems, particularly the protoplanetary disk. X-ray emission may influence: disk ionization, turbulence and viscosity; Jovian planet formation and migration; the production of meteoritic isotopes and melting of meteoritic chondrules; the heating and chemistry of the disk. X-ray surveys are also effective in locating post-T Tauri stars for disk evolution studies.
\end{abstract}

\section{Introduction}

The basic concepts for the formation of low mass stars and their planetary systems have been long known (Jeans 1928). Cold interstellar gas with low thermal pressure undergoes gravitational collapse. Low angular momentum material quickly collects into a protostar in approximate hydrostatic equilibrium, while high angular momentum material enters a Keplerian protoplanetary disk. The actual star formation process and early phases of stellar evolution have proved to be much more complicated than this simple picture. For example, magnetic and turbulent pressures must be considered in addition to thermal pressure in the early stages of collapse; disk material appears to simultaneously accrete onto and be accelerated from the protostar in magnetically collimated flows; complex interactions between gaseous and solid components occur in the disk. But even today, the early evolution of stars and planets is commonly viewed as web of processes involving neutral materials characteristic temperatures $T \sim 10^{0}-10^{3}$ $\mathrm{K}$, and is thus best studied at millimeter, infrared and optical bands. 


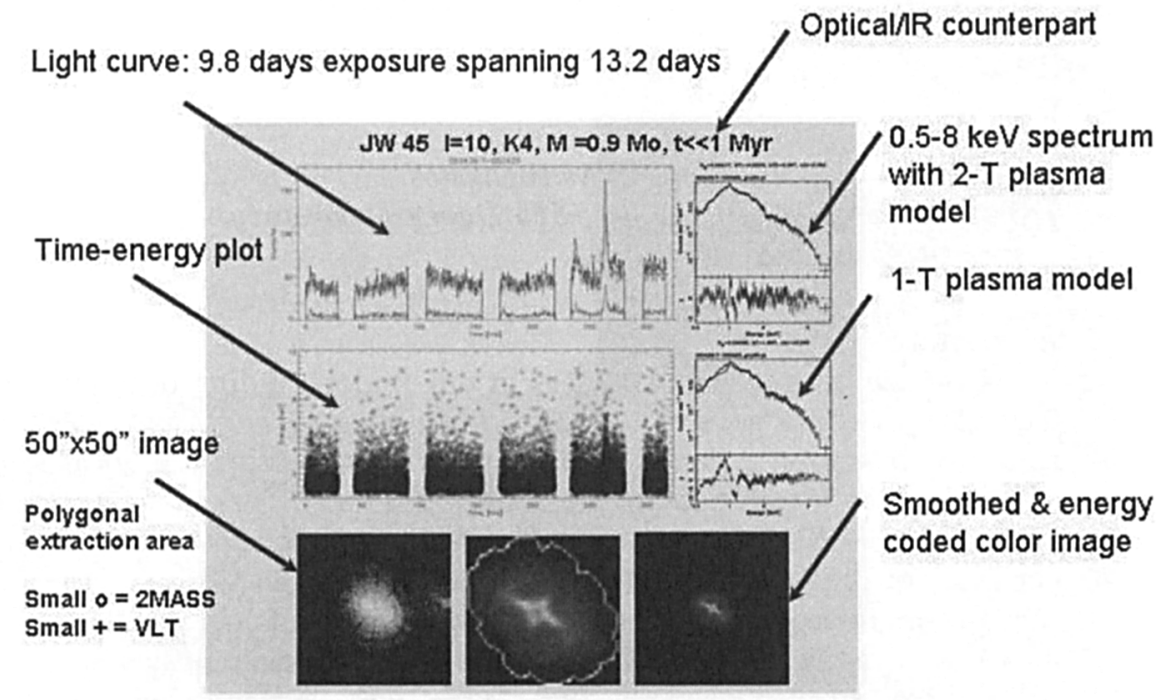

Figure 1. Graphical information obtained for each Chandra Orion Ultradeep Project (COUP) source in the Orion Nebula region. The upper left panels show light curves with energy discrimination. The upper right panels show the $0.5-8 \mathrm{keV}$ spectra with model fits. The lower panels show the neighborhood of the source in the ACIS image.

It is thus perhaps surprising that X-ray telescopes consistently detect thermal emission of $T \sim 10^{7}-10^{8} \mathrm{~K}$ plasmas from nearly all pre-main sequence (PMS) stars ranging from Class I protostars (age $t \sim 10^{5} \mathrm{yr}$ ) to post-T Tauri stars $\left(t \sim 10^{7} \mathrm{yr}\right)$. The X-ray behavior resembles the magnetically active Sun with harder flares superposed on a softer quiescent emission with luminosities $10^{1}-10^{4}$ above contemporary solar flare emission (see review by Feigelson \& Montmerle 1999).

Although PMS X-ray emission is a minor energetic component with $L_{x} / L_{b o l} \sim 10^{-4 \pm 1}$, it is important for three reasons. First, it is the most readily studied tracer of magnetic activity, as the high absorption and distance to most PMS stars hinder measurements ultraviolet study and high resolution optical spectroscopy. Second, it is a useful method for uncovering the PMS population in a disk-unbiased fashion, complementary to infrared techniques that select mainly massive disk systems. PMS X-rays has been detected in thousands of PMS stars out to several kiloparsecs distance, many of them previously uncataloged, and can be seen through $A_{V} \sim 200$ of obscuring material. Third, X-rays can have an important effect on the mostly neutral environment. For example, a $1 \mathrm{keV} \mathrm{X}$-ray traversing a molecular medium will be absorbed in column densities 
$\log N_{H} \sim 21-23 \mathrm{~cm}^{-2}$ from the star, creating roughly 30 molecular ions. Even very low ionization levels can change the gas dynamics by coupling the gas to magnetic fields and can alter the astrochemical balance.

We start here with a brief presentation of some of the findings concerning magnetic activity of PMS stars obtained during the first years of the Chandra $X$-ray Observatory and XMM-Newton mission. The treatment here is very incomplete, as nearly 40 studies have emerged to date. These include studies of young low-mass stars in the Ophiuchus cloud $(d \simeq 140 \mathrm{pc}$; Imanishi et al. 2001a, 2001b, 2002, 2003), Taurus-Auriga clouds ( $d \simeq 140$ pc; Favata et al. 2002, 2003; Bally et al. 2003), Perseus cloud ( $d \simeq 350$ pc; Preibisch \& Zinnecker 2001, 2002; Getman et al. 2002), Orion giant molecular clouds ( $d \simeq 450$ pc; Garmire et al. 2000; Schulz et al. 2001; Pravdo et al. 2001; Tsuboi et al. 2001; Feigelson et al. 2002a, 2002b, 2003a; Flaccomio et al. 2003a, 2003b; Tsujimoto et al. 2002; Grosso et al. 2003; Skinner et al. 2003), older dispersed PMS stars $(d \simeq 50-150$ pc; Kastner et al. 2002; Tsuboi et al. 2003; Stelzer et al. 2003; Feigelson et al. $2003 \mathrm{~b})$ and more massive star forming regions $(d \simeq 1-2 \mathrm{kpc}$; Kohno et al. 2002; Beuther et al. 2002; Evans et al. 2003; Nakajima et al. 2003). Studies performed with the XMM-Newton satellite include Taurus-Auriga (Favata et al. 2003), Perseus (Preibisch 2003), NGC 6530 (Rauw et al. 2002), and NGC 6383 (Rauw et al. 2003).

Chandra observations have been most concentrated on the Orion Nebula Cluster (ONC), the richest PMS stellar cluster within $0.5 \mathrm{kpc}$ with a population of $>2000$ well-characterized stars in a single field (ODell 2002). We give here an early look at the exceptionally deep January 2003 observation of the Orion Nebula region with Chandra's Advanced CCD Imaging Spectrometer (ACIS). The ONC was observed during the first year of Chandra operations with both focal plane detectors. These observations have now been analyzed in detail (Garmire et al. 2000; Feigelson et al. 2002a, 2002b, 2003; Flaccomio et al. 2002, 2003). We obtained $\sim 10$ days exposure over a $\sim 13$ day period in January 2003. This effort, nicknamed the Chandra Orion Ultradeep Project (COUP), provides an extraordinary opportunity to study the magnetic activity of $\sim 1600$ magnetically active PMS stars. COUP is the collective effort of a large collaboration; the science analysis has only begun and a wide range of valuable results are expected to emerge in the next two years.

\section{X-ray flaring in PMS stars}

Figure 1 shows one example of the $\sim 1600$ COUP sources. These outputs are generated with an automated procedure acis_extract developed at Penn State that efficiently reduces and analyzes fields with many point sources (http://www.astro.psu.edu/xray/docs/ae). A tremendous range of X-ray flaring behaviors are seen in the COUP data.

\subsection{Temporal profiles}

Most COUP light curves can be qualitatively described as flares superposed on a quiescent component. Rotational modulations of long-lived asymmetric coronal structures, as have been seen in two main sequence active stars (Marino et al. 2003), rarely dominate the X-ray variations of the Orion stars. 

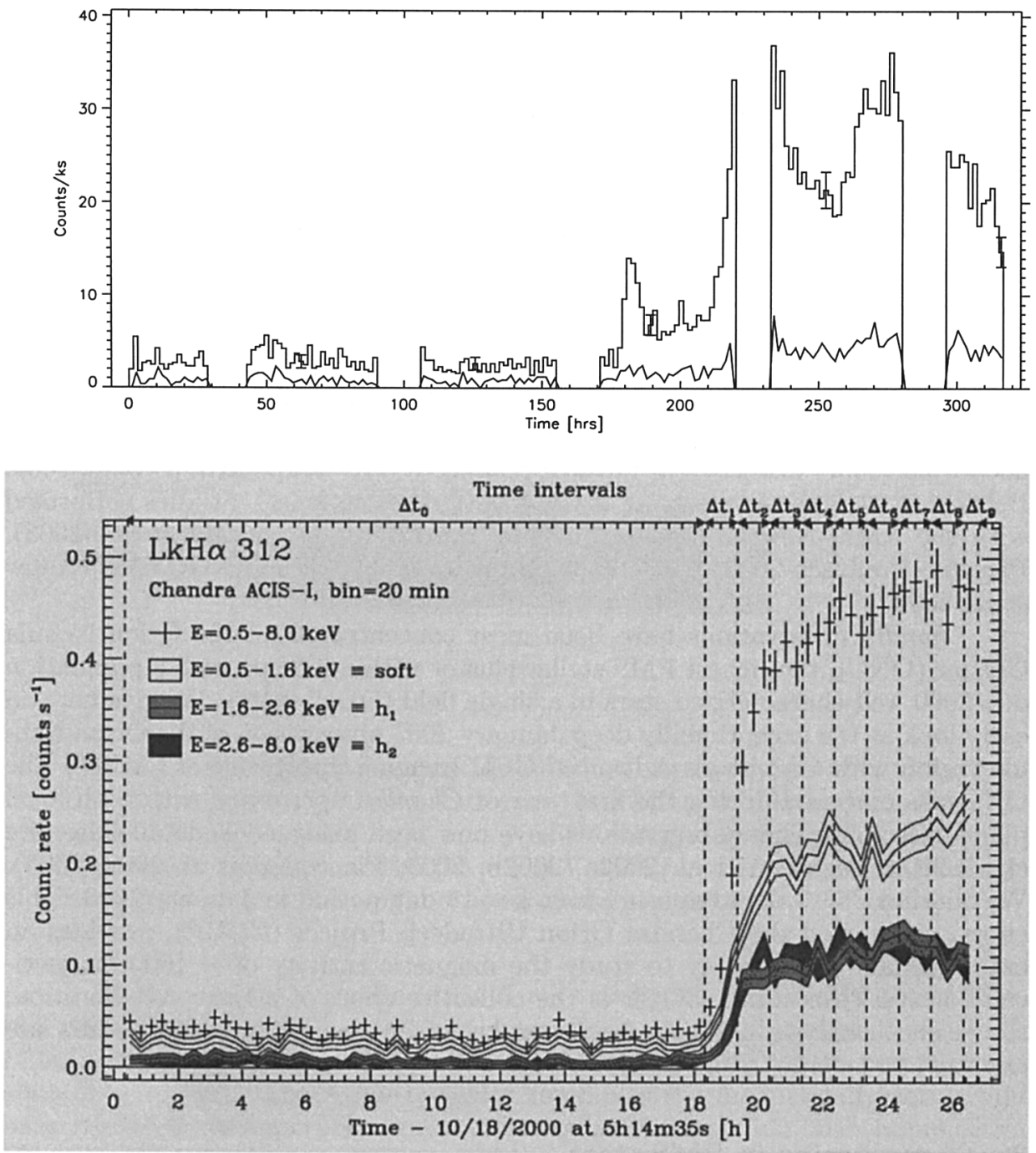

Figure 2. (Top) COUP light curve of multiple flares over 13 days from the obscured Orion Nebula star GMR A = HC 573. The upper line shows Chandra's $0.5-8 \mathrm{keV}$ full band and the lower line shows the soft $0.5-2 \mathrm{keV}$ band (Getman et al. 2003). The events shown here are extracted from an annulus to avoid CCD pileup from this strong source. An uniquely powerful microwave flare occurred during the last data gap around hour 286 (Bower et al. 2003). (Bottom) X-ray light curve in several bands of an unusually powerful flare from the weaklined T Tauri star LkH $\alpha 312$ lying in the NGC 2068 region of the Orion clouds. This is a continuous 1.1 day observation (Grosso et al. 2003). 
Many COUP flares exhibit the classical profiles of many solar flares: sudden (timescale $<1 \mathrm{ks}$ ) rise from a quiescent level to a brief hard peak level followed by an exponential-type decay on timescales hours with monotonic spectral softening (see review of solar flares by Priest \& Forbes 2001). These can be interpreted as a single magnetic reconnection event that fills a single magnetic loop with hot plasma which cools by radiation and/or conduction. Multiple events, some starting during the decay of previous flares, are seen, particularly in the long COUP observation (Figure 2, top panel). This denotes a sequence of reconnection events, perhaps triggered by the new magnetic field structure, and requires complex multipolar field configurations as seen in the Sun. Feigelson et al. (2002b) estimates that PMS solar analogs produce $\log L_{x, p e ~}>29.5 \mathrm{erg} / \mathrm{s}$ flares once every few days.

The main difference between PMS flares and contemporary solar flares is their power. ONC flares have $\log L_{p e ~} k=29-32 \mathrm{erg} / \mathrm{s}$ compared to $24-28$ $\mathrm{erg} / \mathrm{s}$ for contemporary solar flares. A wide range is seen in the durations of PMS flares. Some begin and end within an hour, while others extend for two days or more. The COUP project will seek relationships between flare power, duration and repetition, but such results are not yet available.

\subsection{PMS X-ray spectra}

The ONC sources show hotter plasmas than solar flares: the typical dominant plasma has energy around $2-3 \mathrm{keV}$, and some with dominant plasmas $5-10$ or even $>10 \mathrm{keV}$ (Feigelson et al. 2002a). A strong hardening of the spectrum during flares is seen in Figure 2 . In the bottom panel, a $0.7 \mathrm{M}_{\odot}$ star underwent an unusually powerful flare with $\log L_{x, p e ~}=32.1 \mathrm{erg} / \mathrm{s}$ and $k T_{p e ~}=6 \mathrm{keV}$ after a period of quiescent emission (Grosso et al. 2003). Such behaviors are consistent with the $L_{x}-T$ correlation in solar and stellar flares, and with the $L_{x}$ - age anticorrelation seen in the Sun and solar analogs (Güdel et al. 1997).

Elemental anomalies are often, but not always, present in the stronger flares, most often seen in the neon and iron lines around $1 \mathrm{keV}$. Such phenomena were first seen in high-resolution X-ray spectra of nearby active stars (Brinkman et al. 2001; Drake et al. 2001). An unusual case among Chandra PMS studies is the Class I protostar WL 22 in the Ophiuchus cloud core described by Imanishi et al. (2001). This source is heavily absorbed with $\log N_{H}=22.5 \mathrm{~cm}^{-2}$ so no emission is seen below $2 \mathrm{keV}$, but the unobscured emission is dominated by a calcium line around $3.2 \mathrm{keV}$ and argon line around $3.9 \mathrm{keV}$ implying a $\sim 10$-fold overabundances of these elements (Figure 3, left panel). In contrast, the $\mathrm{LkH} \alpha$ 312 flare shown in Figure 2 (bottom panel) showed no abundances anomalies.

Another rare but important spectral anomaly are the fluorescent lines from cold iron around $6.4 \mathrm{keV}$. It has been seen in two embedded protostars, IRS 7 in the CrA cloud with $A S C A$ (Koyama et al. 1996) and YLW 16A in Ophiuchus with Chandra (Figure 3, right panel; Imanishi et al. 2001a). This arises from the illumination of circumstellar molecular material, either in a disk or envelope, by the PMS X-rays. The fluorescent iron line potentiates a fascinating experiment for future high-throughput X-ray telescopes: reverberation mapping of the complex protostellar and protoplanetary environment on time scales of lighthours (physical scales of tens of AUs) as the $6.4 \mathrm{keV}$ fluorescence responds to PMS X-ray flares. 

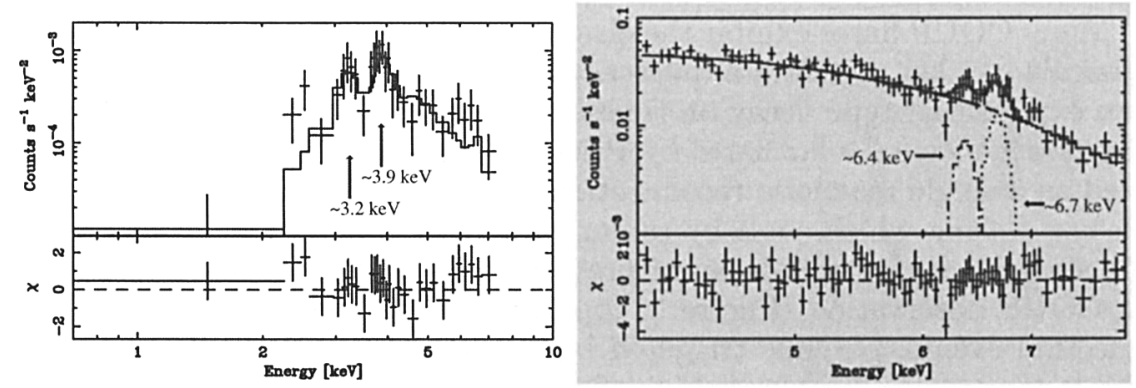

Figure 3. Chandra CCD spectrum of the Ophiuchus cloud Class I protostars WL 22 (left) showing strong Ca and Ar emission lines, and YLW 16A (right) showing $6.4 \mathrm{keV}$ iron fluorescent and $6.7 \mathrm{keV}$ iron plasma emission lines (Imanishi et al. 2001).

Only one PMS star has been sufficiently bright for study with the high resolution grating spectrometer on board Chandra: the classical T Tauri star TW Hya at $d=55 \mathrm{pc}$ (Kastner et al. 2002). Its properties are difficult to interpret. On one hand, the light curve shows a rapid rise and slower fall in flux with $\log L_{x, \text { peak }}=30.3 \mathrm{erg} / \mathrm{s}$ and non-solar abundances of $\mathrm{O}, \mathrm{Ne}$ and Fe. These properties are typical of PMS flares. However, the main plasma component is unusually soft with $k T=0.3 \mathrm{keV}$ and the Ne IX triplet implies a high electron density $\log n=12.8 \mathrm{~cm}^{-3}$. These properties suggest that the emission may be produced in the shock where magnetically collimated disk material slams into the stellar surface.

\subsection{Quiescent emission}

The typical plasma temperatures of quiescent, or inter-flare, PMS X-ray emission in Chandra data are $1-3 \mathrm{keV}$ in contrast to $0.1 \mathrm{keV}$ from the coronal component of the contemporary Sun. Quiescent PMS emission can also reach very high Xray luminosities; e.g. with $\log L_{x, q u i e s}=30.8 \mathrm{erg} / \mathrm{s}$ and $k T=1-3 \mathrm{keV}$ in LkH $\alpha$ 312 (Figure 2, bottom panel, Grosso et al. 2003). Together, these characteristics casts doubt on an association of quiescent with a distributed heating process that powers the solar corona. Indeed, as the principal Chandra imaging detector (ACIS-I) can not reliably detecting photons below $0.5 \mathrm{keV}$, solar-like coronal component in PMS stars are largely inaccessible.

These findings thus support the concept developed for other magnetically active stars that quiescent X-ray emission arises from the superposition of many microflares (Drake et al. 2000, Güdel et al. 2003). However, this interpretation must confront the fact that temporal variations in the quiescent component are often much smaller than the prominent flares, implying an deficit of intermediate luminosity flares.

\section{The absence of a PMS activity-rotation relation}

The principal empirical correlate of magnetic activity in late-type main sequence stars is the rate of rotation (Hartmann \& Noyes 1987). This has served as a key 

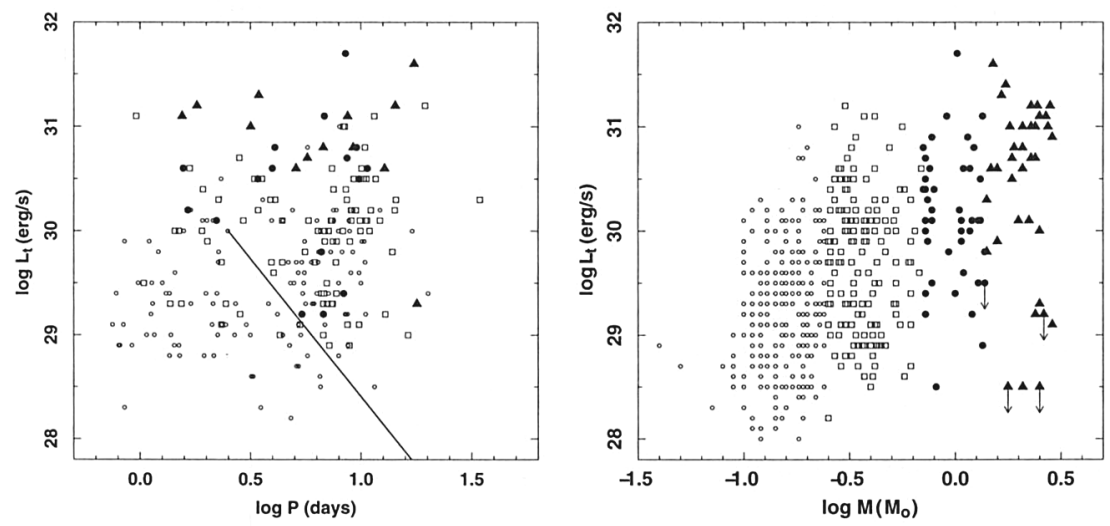

Figure 4. (Left) Plot of X-ray emissivity $v s$. rotational period for 232 Orion Nebula Cluster PMS stars. The line shows the well-established activity-rotation relation for main sequence solar mass stars. (Right) Plot showing a correlation between X-ray emissivity and mass in Orion stars. The symbols denote different mass ranges. From Feigelson et al. (2003)

observational ingredient to the theoretical modelling of magnetic field generation in solar-type stars based on a $\alpha-\Omega$-type dynamo (Schrijver \& Zwaan 2000). Magnetic fields are built up in the thin tachocline layer between the convective and radiative zones, and are transported and twisted through the convective layer to the surface where they produce active regions and undergo violent reconnection flaring. The relationship between X-ray emission and rotation period is very tight, with $\log L_{x}=2.6 \log P$ for solar analogs, until a saturation level around $\log L_{x} / L_{b o l} \simeq-3$ is reached (Guinan et al. 1997, Randich 2000). It is not clear whether this represents a saturation of an internal dynamo process or a saturation of magnetic field emergence or flaring processes at the stellar surface.

Past studies from Einstein Observatory and ROSAT studies gave conflicting evidence concerning the X-ray activity-rotation relation in PMS stars. The new Chandra studies of the Orion Nebula Cluster provide a much larger and more uniform sample of X-ray detected stars with photometrically measured rotation periods (Feigelson et al. 2003, Flaccomio et al. 2003b). The result is clear: no correlation between X-ray emission, measured either as $\log L_{x}$ or $\log L_{x} / L_{b o l}$, and rotation period is present (Figure 4, left panel). Instead, a broad relationship between $\log L_{x}$ and several interlinked stellar properties - bolometric luminosity, mass, stellar surface area and volume - is present (Figure 4, right panel). X-ray emission does not strongly decay with age within the approximately $1 \leq t \leq 10$ Myr range of Orion Nebula Cluster stars.

The explanation for such a dramatic difference between activity in PMS and main sequence stars is not obvious. One reasonable possibility to explain the independence of X-ray emission and rotation is that the dynamo mechanisms are the same but all PMS stars have saturated dynamos. However, this does 


\section{High energy processes in young stellar objects}

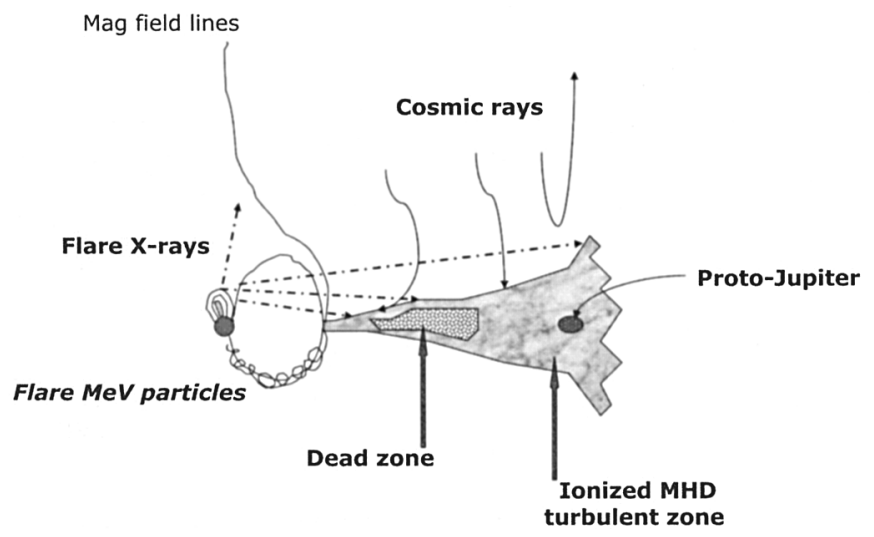

Figure 5. Cartoon illustrating some of the possible links between PMS magnetic activity and the physics of protoplanetary disks and outflows. Ionization by Galactic cosmic rays and PMS stellar X-rays is thought to induce turbulence over portions of the disk. Flare $\mathrm{MeV}$ particles can irradiate disk solids.

not readily explain either why the PMS stars typically show $\log L_{x} / L_{b o l} \simeq-4$ rather than -3 , nor why the $L_{x}-L_{b o l}-M-R$ relations are present.

Another possibility is closer to theoretical interests. Orion stars should be fully (or mostly) convective and thus have no tachocline (or have one that is deeply buried) where magnetic fields strengths are built up. Such stars may generate fields by turbulent motions distributed throughout the convection zone (Durney et al. 1993). Recent calculations of distributed turbulent dynamos suggest that they should be independent of rotation (e.g. Küker \& Stix 2001). But the calculations are not sufficiently advanced to predict the observed $L_{x}-$ $L_{b o l}-M-R$ relations. A potentially valuable line of evidence is the steep drop in $\log L_{x} / L_{b o l}$ for some or all Orion stars above $M \simeq 2-3 \mathrm{M}_{\odot}$. This may represent a transition between a convective dynamo and a (perhaps inefficient) tachocline dynamo (see the discussion of Barnes 2003).

\section{Magnetic flares, circumstellar disks and planet formation}

The most widely discussed effect of PMS X-rays on their environment is the ionization of mainly-neutral molecular gases in protoplanetary disks (see Matsumura \& Pudritz 2003 and the review of earlier work by Glassgold et al. 2000). Together with Galactic cosmic rays, PMS X-rays produce low levels of ionization in the largely neutral disks (Figure 5). The exact effects depend on relative geometries of the reconnecting field and the disk, on the spectrum of the X-rays 
(e.g., $5 \mathrm{keV}$ photons penetrate much deeper than $1 \mathrm{keV}$ photons), and on the uncertain recombination rate in the disk.

But a consensus is emerging that $\mathrm{X}$-ray and cosmic ray ionization are probably sufficient to induce the Balbus-Hawley (magneto-rotational) instability which rapidly develops into full MHD turbulence over large portions of the disk. Calculations indicate the turbulence may penetrate to the midplane in the outer Jovian planetary regions, but a 'dead zone' of neutral laminar flow likely prevails where terrestrial planets form. These conditions conceivably could explain the difference between terrestrial \& Jovian planet formation (Matsumura \& Pudritz 2003). Turbulent eddies in the outer regions produce strongly fluctuating gravitational torques that may slow or stop inward migration of Jovian protoplanets (Winters et al. 2003, Papaloizou et al. 2003, Laughlin et al. 2003). Thus, PMS X-rays may help explain the diversity of extrasolar planetary systems.

Several other effects of PMS magnetic activity on the circumstellar environment have been proposed, though not all are yet convincing.

1. PMS X-rays are likely a principal source of heating and ionization at the base of protostellar Herbig-Haro objects (Shang et al. 2002, Ferro-Fontán et al. 2003). Magnetically collimated Herbig-Haro outflows, together with the closely related molecular bipolar flows, arise from the inner disk regions of virtually all protostellar systems. Such ionization is crucial for the magneto-centrifugal acceleration of material with a planar Keplerian motion to an outward motion perpendicular to the disk.

2. Like powerful solar flares, X-ray flares are likely accompanied by release of $\mathrm{MeV}$ charged particles. $\mathrm{MeV}$ electrons are directly seen in a few PMS stars via their circularly polarized radio gyrosynchrotron radiation (see review by Güdel 2002; Bower et al. 2003). MeV baryons can bombard disk solids and produce unusual isotopes by spallation nuclear reactions. Feigelson et al. (2002) demonstrate that Chandra X-ray flare studies give new observational support to long-standing models of a spallogenic origin for short-lived extinct radionuclides (e.g. ${ }^{10} \mathrm{Be},{ }^{26} \mathrm{Al},{ }^{53} \mathrm{Mn}$ ) in meteoritic calcium-aluminum-rich inclusions (CAIs). The alternative explanation for these unexpected isotopes in ancient meteorites is that the collapse of our solar system was triggered by the injection of stellar material, such as a supernova remnant, enhanced with recently synthesized nuclei (see review by Goswami \& Vanhala 2000). PMS flare particle irradiation can also readily explain correlated track densities and spallogenic ${ }^{21} \mathrm{Ne}$ abundances seen in some meteoritic grains (see review by Woolum \& Hohenberg 1993).

3. X-ray flare may be responsible for the flash heating of chondrules, small melted globules of rock prevalent in many meteorites, or the less common CAIs (Feigelson 1982; Shu et al. 2001). Such models are attractive in their simultaneous explanation of spallogenic irradiation and thermal melting, but must explain how the pebble-sized melted rocks are transported from the vicinity of the star to the Asteroid Belt.

4. Mid- and far-infrared spectroscopy of PMS circumstellar disks have shown some unexpected features which might be explained by X-ray heating. These include a heated layer of gas in outer disk regions (Bary et al. 2003) and the possible presence of aqueous minerals in circumstellar grains (Ceccarelli et al. 2002). X-ray ionization should also alter astrochemical reactions in disks (Aikawa \& Herbst 2001). 
Finally, we note that X-ray imaging surveys are uniquely effective in locating older PMS (post-T Tauri) stars which have lost most of their accretion and disk indicators but still exhibit elevated magnetic activity. Most of the $\sim 5-30$ Myr stars in the solar neighborhood, which are being intensively investigated for disk evolution and planet formation, were originally located by $R O S A T$ (Jayawardhana \& Greene 2001). Some such efforts can be continued with the Chandra and XMM observatories (Stelzer et al. 2003, Feigelson et al. 2003b).

\section{Conclusions}

The study of magnetic activity in pre-main sequence stars is now a mature observational endeavor. Several percent of the time of the Chandra X-ray Observatory is devoted to studies of young stellar clusters; a rich phenomenology of spatial, spectral and temporal X-ray emission is evident.

PMS stars exhibit a high level of high energy processes from magnetic reconnection with a bewildering variety of X-ray flares. Activity is linked to bulk stellar properties $\left(L_{b o l}\right.$, mass, surface area or volume) rather than rotation. This suggests that dynamo processes in deeply convective PMS stars may fundamentally differ from the tachocline dynamo in main sequence stars.

High-energy radiation from magnetic flares affect the circumstellar environment in PMS systems. X-ray emission may influence: disk ionization, turbulence and viscosity; Jovian planet formation and migration; the formation of meteoritic isotopes and chondrules; disk heating and chemistry. X-ray surveys are also effective in locating post-T Tauri stars for disk evolution studies.

The author expresses his gratitude to Thierry Montmerle for presenting this paper at the conference under trying circumstances, and to Kosta Getman for leading the analysis of COUP data. Kosta and Masahiro Tsujimoto provided useful comments on the manuscript. This work is funded by SAO grant GO34009A supporting COUP.

\section{References}

Aikawa, Y. \& Herbst, E. 2001, A\&A, 371, 1107

Bally, J., Feigelson, E., \& Reipurth, B. 2003, ApJ, 584, 843

Barnes, S. A. 2003, ApJ, 586, 464

Bary, J. S., Weintraub, D. A., \& Kastner, J. H. 2003, ApJ, 586, 1136

Beuther, H., Kerp, J., Preibisch, T., Stanke, T., \& Schilke, P. 2002, A\&A, 395, 169

Bower, G. C., Plambeck, R. L., Bolatto, A., McCrady, N., Graham, J. R., de Pater, I., Liu M. C. \& Baganoff, F. K., 2003, ApJ, in press (astro$\mathrm{ph} / 0308277$ )

Brinkman, A. C. et al. 2001, A\&A, 365, L324

Ceccarelli, C., Caux, E., Tielens, A. G. G. M., Kemper, F., Waters, L. B. F. M., \& Phillips, T. 2002, A\&A, 395, L29

Drake, J. J., Peres, G., Orlando, S., Laming, J. M., \& Maggio, A. 2000, ApJ, 545,1074 
Drake, J. J., Brickhouse, N. S., Kashyap, V., Laming, J. M., Huenemoerder,

D. P., Smith, R., \& Wargelin, B. J. 2001, ApJ, 548, L81

Durney, B. R., De Young, D. S., \& Roxburgh, I. W. 1993, Solar Phys., 145, 207

Evans, N. R., Seward, F. D., Krauss, M. I., Isobe, T., Nichols, J., Schlegel, E. M., \& Wolk, S. J. 2003, ApJ, 589, 509

Favata, F., Fridlund, C. V. M., Micela, G., Sciortino, S., \& Kaas, A. A. 2002, A\&A, 386, 204

Favata,F., Giardino, G., Micela, G., Sciortino, S., \& Damiani, F. 2003, A\&A, 403, 187

Feigelson, E. D. 1982, Icarus, 51, 155

Feigelson, E. D. \& Montmerle, T. 1999, ARA\&A, 37, 363

Feigelson, E. D., Broos, P., Gaffney, J. A., Garmire, G., Hillenbrand, L. A., Pravdo, S. H., Townsley, L., \& Tsuboi, Y. 2002a, ApJ, 574, 258

Feigelson, E. D., Garmire, G. P., \& Pravdo, S. H. 2002b, ApJ, 572, 335

Feigelson, E. D., Gaffney, J. A., Garmire, G., Hillenbrand, L. A., \& Townsley, L. 2003a, ApJ, 584, 911

Feigelson, E. D., Lawson, W. A. \& Garmire, G. P., 2003b, ApJ, in press (astro$\mathrm{ph} / 0309059)$

Flaccomio, E., Damiani, F., Micela, G., Sciortino, S., Harnden, F. R., Murray, S. S., \& Wolk, S. J. 2003a, ApJ, 582, 382

Flaccomio, E., Damiani, F., Micela, G., Sciortino, S., Harnden, F. R., Murray, S. S., \& Wolk, S. J. 2003b, ApJ, 582, 398

Ferro-Fontán, C. \& de Castro, A. I. G. 2003, MNRAS, 342, 427

Garmire, G., Feigelson, E. D., Broos, P., Hillenbrand, L. A., Pravdo, S. H., Townsley, L., \& Tsuboi, Y. 2000, AJ, 120, 1426

Getman, K. V., Feigelson, E. D., Townsley, L., Bally, J., Lada, C. J., \& Reipurth, B. 2002, ApJ, 575, 354

Getman, K. V., Feigelson, E. D., Garmire, G., Murray, S. S. \& Harnden, F. R., 2003, IAU Circ. 8068, 2

Glassgold, A. E., Feigelson, E. D., \& Montmerle, T. 2000, in Protostars and Planets IV, 429

Goswami, J. N. \& Vanhala, H. A. T. 2000, in Protostars and Planets IV, 963

Güdel, M., Guinan, E. F., \& Skinner, S. L. 1997, ApJ, 483, 947

Güdel, M., Audard, M., Kashyap, V. L., Drake, J. J., \& Guinan, E. F. 2003, ApJ, 582, 423

Güdel, M. 2002, ARA\&A, 40, 217

Hartmann, L. W. \& Noyes, R. W. 1987, ARA\&A, 25, 271

Imanishi, K., Koyama, K., \& Tsuboi, Y. 2001a, ApJ, 557, 747

Imanishi, K., Tsujimoto, M., \& Koyama, K. 2001b, ApJ, 563, 361

Imanishi, K., Tsujimoto, M., \& Koyama, K. 2002, ApJ, 572, 300

Imanishi, K., Nakajima, H., Tsujimoto, M., Koyama, K., \& Tsuboi, Y. 2003, PASJ, 55, 653 
Jayawardhana, R. \& Greene, T. (eds.) 2001, Young Stars Near Earth: Progress and Prospects, ASP Conf. 244

Jeans, J. H. 1928, Astronomy and Cosmogony, Cambridge Univ. Press

Kastner, J. H., Huenemoerder, D. P., Schulz, N. S., Canizares, C. R., \& Weintraub, D. A. 2002, ApJ, 567, 434

Kohno, M., Koyama, K., \& Hamaguchi, K. 2002, ApJ, 567, 423

Küker, M. \& Stix, M. 2001, A\&A, 366, 668

Laughlin, G., Steinacker, A. \& Adams, F. 2003, ApJ, submitted, astro-ph/0308406 Marino, A., Micela, G., Peres, G., \& Sciortino, S. 2003, A\&A, 407, L63

Matsumura, S. \& Purditz, R. E., 2003, ApJ, in press, astro-ph/0308039

Nakajima, H., Imanishi, K., Takagi, S., Koyama, K., \& Tsujimoto, M. 2003, PASJ, 55, 635

Papaloizou, J. C., Nelson, R. P. \& Snellgrove, M. D. 2003, MNRAS, submitted, astro-ph/0308351

Pravdo, S. H., Feigelson, E. D., Garmire, G., Maeda, Y., Tsuboi, Y., \& Bally, J. 2001, Nature, 413, 708

Preibisch, T. \& Zinnecker, H. 2001, AJ, 122, 866

Preibisch, T. \& Zinnecker, H. 2002, AJ, 123, 1613

Preibisch, T. 2003, A\&A, 401, 543

Rauw, G., Nazé, Y., Gosset, E., Stevens, I. R., Blomme, R., Corcoran, M. F., Pittard, J. M., \& Runacres, M. C. 2002, A\&A, 395, 499

Rauw, G., De Becker, M., Gosset, E., Pittard, J. M., \& Stevens, I. R. 2003, A\&A, 407, 925

Randich, S. 2000, in Stellar Clusters and Associations: Convection, Rotation, and Dynamos, ASP Conf. 198, 401

Schrijver, C. J. \& Zwaan, C. 2000, Solar and stellar magnetic activity, Cambridge Univ. Press

Schulz, N. S., Canizares, C., Huenemoerder, D., Kastner, J. H., Taylor, S. C., \& Bergstrom, E. J. 2001, ApJ, 549, 441

Shang, H., Glassgold, A. E., Shu, F. H., \& Lizano, S. 2002, ApJ, 564, 853

Shu, F. H., Shang, H., Gounelle, M., Glassgold, A. E., \& Lee, T. 2001, ApJ, 548,1029

Skinner, S., Gagne, M., \& Belzer, E. 2003, astro-ph/0306566

Stelzer, B., Huélamo, N., Hubrig, S., Zinnecker, H., \& Micela, G. 2003, A\&A, 407, 1067

Tsuboi, Y., Koyama, K., Hamaguchi, K., Tatematsu, K., Sekimoto, Y., Bally, J., \& Reipurth, B. 2001, ApJ, 554, 734

Tsuboi, Y., Maeda, Y., Feigelson, E. D., Garmire, G. P., Chartas, G., Mori, K., \& Pravdo, S. H. 2003, ApJ, 587, L51

Tsujimoto, M., Koyama, K., Tsuboi, Y., Goto, M., \& Kobayashi, N. 2002, ApJ, $566,974 \mathrm{r}$

Winters, W. F., Balbus, S. A., \& Hawley, J. F. 2003, ApJ, 589, 543

Woolum, D. S. \& Hohenberg, C. 1993, in Protostars and Planets III, 903 